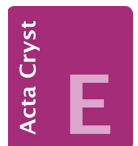

CRYSTALLOGRAPHIC COMMUNICATIONS

ISSN 2056-9890

\section{Corrigenda for three related articles}

\section{P. L. Nilantha Lakshman}

Department of Food Science and Technology, University of Ruhuna, Mapalana, Kamburupitiya 81100, Sri Lanka. *Correspondence e-mail:

plakshmannilantha@ymail.com

Received 20 February 2015; accepted 20 February 2015

The schemes and chemical names are corrected in three related papers: Vishnupriya, Suresh, Bharkavi et al. [Acta Cryst. (2014), E70, o968-0969], Vishnupriya, Suresh, Gunasekaran et al. [Acta Cryst. (2014), E70, o978], and Vishnupriya, Suresh, Sakthi et al. [Acta Cryst. (2014), E70, o1120-o1121].

In the paper by Vishnupriya, Suresh, Bharkavi et al. (2014), the chemical name in the title should be given as "4-(2bromophenyl)-2-(1H-indol-3-yl)-6-(thiophen-2-yl)pyridine-3carbonitrile' and the correct scheme is shown below.<smiles>N#Cc1c(-c2c[nH]c3ccccc23)cc(-c2cccs2)nc1-c1ccccc1Br</smiles>

In the paper by Vishnupriya, Suresh, Gunasekaran et al. (2014), the chemical name in the title should be given as "4-(4chlorophenyl)-2-(1H-indol-3-yl)-6-phenylpyridine-3-carbonitrile' and the correct scheme is shown below.<smiles>N#Cc1c(-c2ccc(Cl)cc2)cc(-c2ccccc2)nc1-c1c[nH]c2ccccc12</smiles>

In the paper by Vishnupriya, Suresh, Sakthi et al. (2014), the chemical name in the title should be given as ' 2 -( $1 H$-indol-3yl)-4-(4-methoxyphenyl)-6-phenylpyridine-3-carbonitrile' and the correct scheme is shown below.<smiles>COc1ccc(-c2cc(-c3ccccc3)nc(-c3c[nH]c4ccccc34)c2C#N)cc1</smiles>

\section{References}

Vishnupriya, R., Suresh, J., Bharkavi, S., Perumal, S. \& Lakshman, P. L. N. (2014). Acta Cryst. E70, o968-0969.

Vishnupriya, R., Suresh, J., Gunasekaran, P., Perumal, S. \& Lakshman, P. L. N. (2014). Acta Cryst. E70, o978.

Vishnupriya, R., Suresh, J., Sakthi, M., Perumal, S. \& Lakshman, P. L. N. (2014). Acta Cryst. E70, o1120-o1121. 


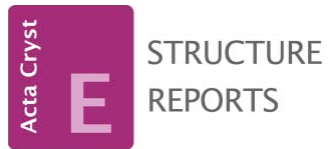

OPEN $\curvearrowright$ ACCESS

ISSN 1600-5368

\section{Crystal structure of 4-(1H-indol-3-yl)-2- (4-methoxyphenyl)-6-phenylpyridine-3- carbonitrile}

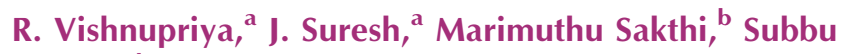
Perumal $^{\mathrm{b}}$ and P. L. Nilantha Lakshman ${ }^{\mathrm{c} *}$

aDepartment of Physics, The Madura College, Madurai 625 011, India, bepartment of Organic Chemistry, School of Chemistry, Madurai Kamaraj University, Madurai 625 021, India, and ${ }^{\mathrm{c}}$ Department of Food Science and Technology, University of Ruhuna, Mapalana, Kamburupitiya 81100, Sri Lanka. *Correspondence e-mail: plakshmannilantha@ymail.com

Received 4 September 2014; accepted 7 September 2014

Edited by W. T. A. Harrison, University of Aberdeen, Scotland

In the title compound, $\mathrm{C}_{27} \mathrm{H}_{19} \mathrm{~N}_{3} \mathrm{O}$, the dihedral angles between the plane of the pyridine ring and those of the indole (r.m.s. deviation $=0.018 \AA$ ), phenyl and methoxybenzene substituents are $33.60(6), 25.28(7)$ and $49.31(7)^{\circ}$, respectively. The $\mathrm{N}$ atom of the carbonitrile group is significantly displaced [0.288 (2) $\AA$ ] from the plane of the pyridine ring, perhaps due to steric crowding. In the crystal, inversion dimers linked by pairs of $\mathrm{N}-\mathrm{H} \cdots \mathrm{N}_{\mathrm{n}}(\mathrm{n}=$ nitrile $)$ hydrogen bonds generate $R_{2}^{2}(16)$ loops. Aromatic $\pi-\pi$ stacking [centroid-centroid separation $=3.6906(7) \AA$ ] and very weak $\mathrm{C}-\mathrm{H} \cdots \pi$ interactions are also observed".

Keywords: crystal structure; pyridine-3-carbonitrile; heterocyclic compounds; hydrogen bonding.

CCDC reference: 1023204

\section{Related literature}

For the use of 2-amino-3-cyanopyridines as intermediates in the preparation of heterocyclic compounds, see: Shishoo et al. (1983).<smiles>COc1ccc(-c2nc(-c3ccccc3)cc(-c3c[nH]c4ccccc34)c2C#N)cc1</smiles>

\section{Experimental}

\subsection{Crystal data}

\section{$\mathrm{C}_{27} \mathrm{H}_{19} \mathrm{~N}_{3} \mathrm{O}$}

$M_{r}=401.45$

Orthorhombic, $\mathrm{Pbca}$

$a=15.7102(5) \AA$

$b=10.7491(3) \AA$

$c=24.3648(7) \AA$

\subsection{Data collection}

Bruker Kappa APEXII diffractometer

Absorption correction: multi-scan (SADABS; Sheldrick, 1996)

$T_{\min }=0.976, T_{\max }=0.980$

\subsection{Refinement}

$R\left[F^{2}>2 \sigma\left(F^{2}\right)\right]=0.039$

$w R\left(F^{2}\right)=0.104$

$S=1.01$

4486 reflections

$V=4114.5(2) \AA^{3}$

$Z=8$

Mo $K \alpha$ radiation

$\mu=0.08 \mathrm{~mm}^{-1}$

$T=293 \mathrm{~K}$

$0.30 \times 0.28 \times 0.25 \mathrm{~mm}$

Table 1

Hydrogen-bond geometry $\left(\AA{ }^{\circ}\right)$.

$C g 1$ is the centroid of the pyrrole ring.

\begin{tabular}{lllll}
\hline$D-\mathrm{H} \cdots A$ & $D-\mathrm{H}$ & $\mathrm{H} \cdots A$ & $D \cdots A$ & $D-\mathrm{H} \cdots A$ \\
\hline $\mathrm{N} 3-\mathrm{H} 3 \cdots \mathrm{N} 2^{\mathrm{i}}$ & 0.86 & 2.15 & $2.9693(19)$ & 159 \\
$\mathrm{C} 32-\mathrm{H} 32 \cdots C g 1^{\text {ii }}$ & 0.93 & 3.00 & $3.9157(19)$ & 170
\end{tabular}

Symmetry codes: (i) $-x,-y+1,-z+2$; (ii) $-x+1,-y+1,-z$.

Data collection: APEX2 (Bruker, 2004); cell refinement: SAINT (Bruker, 2004); data reduction: $S A I N T$; program(s) used to solve structure: SHELXS97 (Sheldrick, 2008); program(s) used to refine structure: SHELXL97 (Sheldrick, 2008); molecular graphics: PLATON (Spek, 2009); software used to prepare material for publication: SHELXL97.

\section{Acknowledgements}

JS and RV thank the management of Madura College for their encouragement and support. SP thanks the Department of Science and Technology, New Delhi, for a major research project (SR/S1/OC/-50/2011) and the University Grants 


\section{data reports}

Commission, New Delhi, for the award of a BSR Faculty Fellowship

Supporting information for this paper is available from the IUCr electronic archives (Reference: HB7280).

\section{References}

Bruker (2004). APEX2 and SAINT. Bruker AXS Inc., Madison, Wisconsin, USA.

Sheldrick, G. M. (1996). SADABS. University of Göttingen, Germany.

Sheldrick, G. M. (2008). Acta Cryst. A64, 112-122.

Shishoo, C. J., Devani, M. B., Bhadti, V. S., Ananthan, S. \& Ullas, G. V. (1983). Tetrahedron Lett. pp. 4611-4612.

Spek, A. L. (2009). Acta Cryst. D65, 148-155. 


\section{supporting information}

Acta Cryst. (2014). E70, o1120-o1121 [doi:10.1107/S1600536814020170]

\section{Crystal structure of 4-(1H-indol-3-yl)-2-(4-methoxyphenyl)-6-phenylpyridine-3- carbonitrile}

\section{R. Vishnupriya, J. Suresh, Marimuthu Sakthi, Subbu Perumal and P. L. Nilantha Lakshman \\ S1. Comment}

Derivatives of 3-cyanopyridine are important and useful intermediates in preparing a varity of heterocyclic compounds (Shishoo et al., 1983). Therefore, the synthesis of 3-cyanopyridine derivatives attracts much interest in organic chemistry. It was in this context that the title compound, was investigated.

The deviation of the nitrile atoms (C41,N2) from the mean plane of the pyridine ring system is -0.1497 (1) $\AA$ and $-0.2886(5) \AA$. The shortening of the $\mathrm{C}-\mathrm{N}$ distances [1.337 (3) and $1.341 \AA]$ and the opening of the $\mathrm{N} 1-\mathrm{C} 11-\mathrm{C} 10$ angle [121.15 (2) ${ }^{\circ}$ ] may be attributed to the size of the substituent at C1, correlating well with the values observed in the orthosubstituted derivative. The dihedral angle between the pseudo-axial phenyl substituent and the plane of the pyridine ring is $69.13(8)^{\circ}$.

The crystal structure features an $\mathrm{N}-\mathrm{H} \cdots \mathrm{N}$ interaction between inverse related molecules generating a graph set ring motif $R_{2}^{2}$ (16) which are linked into chains through $\mathrm{C}-\mathrm{H} \cdots C g 1$ interation $(\mathrm{Cg} 1$ is the centroid of the pyrrole ring of the indole moiety) and by $\pi \cdots \pi$ stacking interaction involving adjacent pyridine rings of the symmetry related molecule at (1$X, 1-Y,-Z)$, with a centroid-to-centroid distance of 3.6906 (7) $\AA$ (Fig 2).

\section{S2. Experimental}

A mixture of 3-(1H-indol-3-yl)-3-oxopropanenitrile 1 (1 mmol), 4,4,4-trifluoro-1- phenylbutane-1,3-dione 2 (1 mmol) and 4-methoxy benzaldehyde $3(1 \mathrm{mmol})$ in the presence of ammonium acetate $(400 \mathrm{mmol})$ under solvent-free condition was heated at $110^{\circ} \mathrm{C}$ for $7 \mathrm{~h}$. After completion of the reaction (TLC), the reaction mixture was poured into water and extracted with dichloromethane. After removal of the solvent, the residue was chromatographed over $\sigma$ ilica gel (230-400 mesh) using petroleum ether-ethyl acetate mixture $(7: 3 v / v)$, which afforded the pure compound.

Melting point: $265^{\circ} \mathrm{C}$, Yield: $72 \%$.

\section{S3. Refinement}

$\mathrm{H}$ atoms were placed at calculated positions and allowed to ride on their carrier atoms with $\mathrm{C}-\mathrm{H}=0.93-0.98 \AA$ and with $U_{\text {iso }}=1.2 U_{\text {eq }}(\mathrm{C}, \mathrm{N})$ for $\mathrm{N}, \mathrm{CH}_{2}$ and $\mathrm{CH}$ atoms and $U_{\text {iso }}=1.5 U_{\mathrm{eq}}(\mathrm{C})$ for $\mathrm{CH}_{3}$ atoms. 


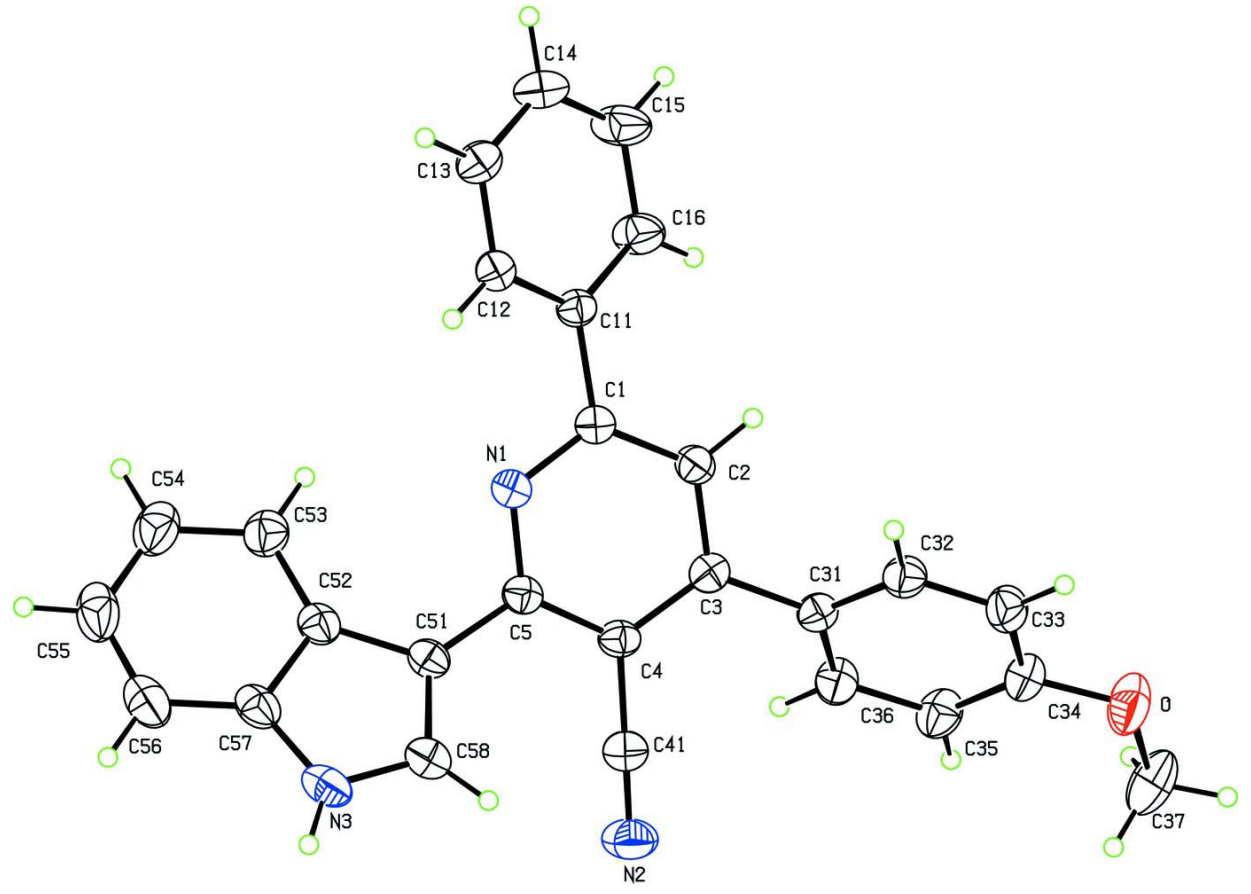

\section{Figure 1}

The molecular structure of compound showing $30 \%$ probability displacement ellipsoids. 


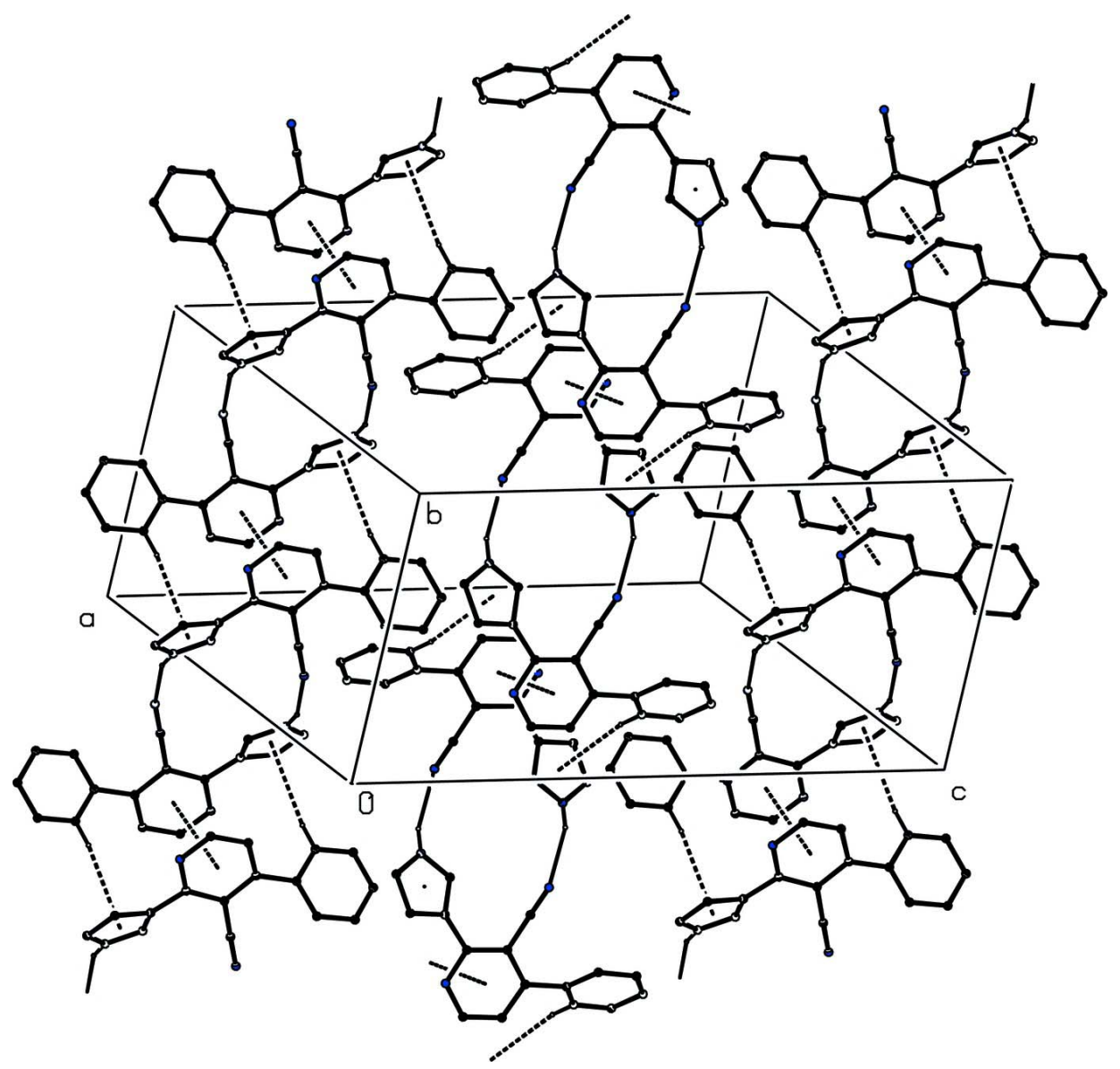

Figure 2

partial packing view of the compound showing molecules interconnected through a $\mathrm{C}-\mathrm{H} \cdots \pi$ and $\pi \cdots \pi$ stacking interaction (dotted lines; symmetry code: (i) $(1-x, 1-y,-z)$

\section{4-(1H-Indol-3-yl)-2-(4-methoxyphenyl)-6-phenylpyridine-3-carbonitrile}

Crystal data

$\mathrm{C}_{27} \mathrm{H}_{19} \mathrm{~N}_{3} \mathrm{O}$

$M_{r}=401.45$

Orthorhombic, $\mathrm{Pbca}$

Hall symbol: -P $2 \mathrm{ac} 2 \mathrm{ab}$

$a=15.7102(5) \AA$

$b=10.7491$ (3) $\AA$

$c=24.3648(7) \AA$

$V=4114.5(2) \AA^{3}$

$Z=8$

\section{Data collection}

\section{Bruker Kappa APEXII}

diffractometer

Radiation source: fine-focus sealed tube

Graphite monochromator

Detector resolution: 0 pixels $\mathrm{mm}^{-1}$

$\omega$ and $\varphi$ scans
$F(000)=1680$

$D_{\mathrm{x}}=1.296 \mathrm{Mg} \mathrm{m}^{-3}$

Mo $K \alpha$ radiation, $\lambda=0.71073 \AA$

Cell parameters from 2000 reflections

$\theta=2-27^{\circ}$

$\mu=0.08 \mathrm{~mm}^{-1}$

$T=293 \mathrm{~K}$

Block, colourless

$0.30 \times 0.28 \times 0.25 \mathrm{~mm}$

Absorption correction: multi-scan

(SADABS; Sheldrick, 1996)

$T_{\text {min }}=0.976, T_{\max }=0.980$

27554 measured reflections

4486 independent reflections

3331 reflections with $I>2 \sigma(I)$ 


$$
\begin{aligned}
& R_{\text {int }}=0.025 \\
& \theta_{\max }=27.0^{\circ}, \theta_{\min }=2.1^{\circ} \\
& h=-20 \rightarrow 20
\end{aligned}
$$

\section{Refinement}

Refinement on $F^{2}$

Least-squares matrix: full

$R\left[F^{2}>2 \sigma\left(F^{2}\right)\right]=0.039$

$w R\left(F^{2}\right)=0.104$

$S=1.01$

4486 reflections

282 parameters

0 restraints

Primary atom site location: structure-invariant direct methods

Secondary atom site location: difference Fourier map

\section{Special details}

Geometry. All e.s.d.'s (except the e.s.d. in the dihedral angle between two 1.s. planes) are estimated using the full covariance matrix. The cell e.s.d.'s are taken into account individually in the estimation of e.s.d.'s in distances, angles and torsion angles; correlations between e.s.d.'s in cell parameters are only used when they are defined by crystal symmetry. An approximate (isotropic) treatment of cell e.s.d.'s is used for estimating e.s.d.'s involving l.s. planes.

Refinement. Refinement of $F^{2}$ against ALL reflections. The weighted $R$-factor $w R$ and goodness of fit $S$ are based on $F^{2}$, conventional $R$-factors $R$ are based on $F$, with $F$ set to zero for negative $F^{2}$. The threshold expression of $F^{2}>\sigma\left(F^{2}\right)$ is used only for calculating $R$-factors $(\mathrm{gt})$ etc. and is not relevant to the choice of reflections for refinement. $R$-factors based on $F^{2}$ are statistically about twice as large as those based on $F$, and $R$ - factors based on ALL data will be even larger.

Fractional atomic coordinates and isotropic or equivalent isotropic displacement parameters $\left(\AA^{2}\right)$

\begin{tabular}{lllll}
\hline & $x$ & $y$ & $z$ & $U_{\text {iso }} * / U_{\text {eq }}$ \\
\hline C1 & $0.11716(8)$ & $1.07304(12)$ & $0.96916(5)$ & $0.0400(3)$ \\
C2 & $0.14114(9)$ & $1.06339(12)$ & $1.02373(5)$ & $0.0436(3)$ \\
H2 & 0.1574 & 1.1344 & 1.0428 & $0.052^{*}$ \\
C3 & $0.14124(8)$ & $0.94978(12)$ & $1.05007(5)$ & $0.0416(3)$ \\
C4 & $0.11465(8)$ & $0.84683(11)$ & $1.01907(5)$ & $0.0416(3)$ \\
C5 & $0.08413(8)$ & $0.86400(11)$ & $0.96531(5)$ & $0.0407(3)$ \\
C11 & $0.12231(8)$ & $1.19255(12)$ & $0.93922(5)$ & $0.0402(3)$ \\
C12 & $0.07016(9)$ & $1.21553(12)$ & $0.89470(5)$ & $0.0465(3)$ \\
H12 & 0.0316 & 1.1552 & 0.8833 & $0.056^{*}$ \\
C13 & $0.07492(11)$ & $1.32728(14)$ & $0.86716(6)$ & $0.0559(4)$ \\
H13 & 0.0391 & 1.3420 & 0.8375 & $0.067 *$ \\
C14 & $0.13180(11)$ & $1.41679(14)$ & $0.88303(7)$ & $0.0605(4)$ \\
H14 & 0.1350 & 1.4917 & 0.8641 & $0.073 *$ \\
C15 & $0.18372(11)$ & $1.39515(15)$ & $0.92690(8)$ & $0.0711(5)$ \\
H15 & 0.2223 & 1.4558 & 0.9380 & $0.085^{*}$ \\
C16 & $0.17937(10)$ & $1.28396(14)$ & $0.95483(7)$ & $0.0614(4)$ \\
H16 & 0.2152 & 1.2701 & 0.9846 & $0.074 *$ \\
C31 & $0.16647(9)$ & $0.94381(12)$ & $1.10856(5)$ & $0.0430(3)$ \\
C32 & $0.13218(10)$ & $1.03013(13)$ & $1.14465(6)$ & $0.0515(4)$ \\
H32 & 0.0935 & 1.0886 & 1.1317 & $0.062^{*}$
\end{tabular}

Hydrogen site location: inferred from

where $P=\left(F_{\mathrm{o}}^{2}+2 F_{\mathrm{c}}^{2}\right) / 3$

$\Delta \rho_{\max }=0.15$ e $\AA^{-3}$

$\Delta \rho_{\min }=-0.16$ e $\AA^{-3}$

Extinction correction: SHELXL97 (Sheldrick, neighbouring sites

$(\Delta / \sigma)_{\max }=0.001$

Extinction coefficient: 0.0027 (4)
$\mathrm{H}$-atom parameters constrained

$k=-13 \rightarrow 8$
$l=-31 \rightarrow 31$ 


$\begin{array}{lllll}\text { C33 } & 0.15425(11) & 1.03077(14) & 1.19904(6) & 0.0576(4) \\ \text { H33 } & 0.1301 & 1.0888 & 1.2227 & 0.069^{*} \\ \text { C34 } & 0.21220(10) & 0.94553(14) & 1.21888(6) & 0.0547(4) \\ \text { C35 } & 0.24701(10) & 0.85934(15) & 1.18375(6) & 0.0586(4) \\ \text { H35 } & 0.2862 & 0.8015 & 1.1968 & 0.070^{*} \\ \text { C36 } & 0.22377(10) & 0.85875(14) & 1.12909(6) & 0.0532(4) \\ \text { H36 } & 0.2473 & 0.7997 & 1.1057 & 0.064^{*} \\ \text { C37 } & 0.28200(13) & 0.8619(2) & 1.29741(7) & 0.0858(6) \\ \text { H37A } & 0.2574 & 0.7814 & 1.2910 & 0.129^{*} \\ \text { H37B } & 0.2861 & 0.8763 & 1.3362 & 0.129^{*} \\ \text { H37C } & 0.3378 & 0.8651 & 1.2814 & 0.129^{*} \\ \text { C41 } & 0.11876(9) & 0.72404(13) & 1.04200(6) & 0.0492(3) \\ \text { C51 } & 0.04591(9) & 0.76538(12) & 0.93237(6) & 0.0441(3) \\ \text { C52 } & 0.04502(9) & 0.75788(12) & 0.87349(6) & 0.0462(3) \\ \text { C53 } & 0.08391(10) & 0.82422(15) & 0.83125(6) & 0.0572(4) \\ \text { H53 } & 0.1182 & 0.8925 & 0.8390 & 0.069^{*} \\ \text { C54 } & 0.07081(13) & 0.78724(17) & 0.77795(7) & 0.0721(5) \\ \text { H54 } & 0.0967 & 0.8311 & 0.7496 & 0.087^{*} \\ \text { C55 } & 0.01956(14) & 0.68547(18) & 0.76559(8) & 0.0784(6) \\ \text { H55 } & 0.0120 & 0.6623 & 0.7291 & 0.094^{*} \\ \text { C56 } & -0.01989(12) & 0.61900(15) & 0.80601(8) & 0.0704(5) \\ \text { H56 } & -0.0543 & 0.5511 & 0.7977 & 0.085^{*} \\ \text { C57 } & -0.00692(10) & 0.65614(13) & 0.85977(7) & 0.0533(4) \\ \text { C58 } & -0.00419(10) & 0.66969(13) & 0.95069(6) & 0.0529(4) \\ \text { H58 } & -0.0145 & 0.6513 & 0.9874 & 0.063^{*} \\ \text { N1 } & 0.08729(7) & 0.97572(9) & 0.94093(4) & 0.0419(3) \\ \text { N2 } & 0.12503(10) & 0.62657(12) & 1.05973(6) & 0.0694(4) \\ \text { N3 } & -0.03648(9) & 0.60601(11) & 0.90767(6) & 0.0597(4) \\ \text { H3 } & -0.0704 & 0.5435 & 0.9101 & 0.072^{*} \\ \text { O } & 0.22989(9) & 0.95450(12) & 1.27328(4) & 0.0808(4)\end{array}$

Atomic displacement parameters $\left(\AA^{2}\right)$

\begin{tabular}{lllllll}
\hline & $U^{11}$ & $U^{22}$ & $U^{33}$ & $U^{12}$ & $U^{13}$ & $U^{23}$ \\
\hline C1 & $0.0366(7)$ & $0.0401(7)$ & $0.0433(7)$ & $0.0005(5)$ & $0.0008(5)$ & $0.0017(5)$ \\
C2 & $0.0461(8)$ & $0.0390(7)$ & $0.0457(7)$ & $-0.0014(6)$ & $-0.0031(6)$ & $-0.0012(6)$ \\
C3 & $0.0385(7)$ & $0.0436(7)$ & $0.0427(7)$ & $0.0036(5)$ & $0.0014(5)$ & $0.0027(6)$ \\
C4 & $0.0398(7)$ & $0.0374(7)$ & $0.0477(7)$ & $0.0027(5)$ & $0.0046(6)$ & $0.0030(6)$ \\
C5 & $0.0383(7)$ & $0.0384(7)$ & $0.0453(7)$ & $0.0021(5)$ & $0.0043(6)$ & $-0.0007(6)$ \\
C11 & $0.0390(7)$ & $0.0396(7)$ & $0.0420(7)$ & $0.0000(5)$ & $0.0025(5)$ & $0.0025(5)$ \\
C12 & $0.0513(8)$ & $0.0437(7)$ & $0.0444(7)$ & $-0.0006(6)$ & $-0.0023(6)$ & $-0.0008(6)$ \\
C13 & $0.0626(10)$ & $0.0533(8)$ & $0.0518(9)$ & $0.0050(7)$ & $-0.0070(7)$ & $0.0100(7)$ \\
C14 & $0.0608(10)$ & $0.0472(8)$ & $0.0736(11)$ & $0.0003(7)$ & $0.0047(8)$ & $0.0200(8)$ \\
C15 & $0.0608(10)$ & $0.0561(9)$ & $0.0963(13)$ & $-0.0219(8)$ & $-0.0149(9)$ & $0.0197(9)$ \\
C16 & $0.0568(9)$ & $0.0567(9)$ & $0.0706(10)$ & $-0.0151(7)$ & $-0.0196(8)$ & $0.0164(8)$ \\
C31 & $0.0436(7)$ & $0.0424(7)$ & $0.0429(7)$ & $0.0017(6)$ & $0.0001(6)$ & $0.0037(6)$ \\
C32 & $0.0586(9)$ & $0.0455(8)$ & $0.0504(8)$ & $0.0109(7)$ & $-0.0023(7)$ & $0.0032(6)$ \\
C33 & $0.0707(10)$ & $0.0544(9)$ & $0.0477(8)$ & $0.0103(8)$ & $0.0017(7)$ & $-0.0055(7)$
\end{tabular}




\begin{tabular}{lllllll} 
C34 & $0.0593(9)$ & $0.0642(9)$ & $0.0407(7)$ & $0.0013(8)$ & $-0.0038(7)$ & $0.0020(7)$ \\
C35 & $0.0568(9)$ & $0.0660(10)$ & $0.0528(8)$ & $0.0164(7)$ & $-0.0072(7)$ & $0.0068(7)$ \\
C36 & $0.0550(9)$ & $0.0555(8)$ & $0.0490(8)$ & $0.0152(7)$ & $-0.0012(7)$ & $-0.0019(7)$ \\
C37 & $0.0742(12)$ & $0.1313(17)$ & $0.0517(10)$ & $0.0134(12)$ & $-0.0137(9)$ & $0.0174(11)$ \\
C41 & $0.0482(8)$ & $0.0456(8)$ & $0.0539(8)$ & $0.0005(6)$ & $0.0004(6)$ & $0.0042(6)$ \\
C51 & $0.0444(7)$ & $0.0367(7)$ & $0.0511(8)$ & $0.0035(6)$ & $-0.0004(6)$ & $-0.0023(6)$ \\
C52 & $0.0466(7)$ & $0.0389(7)$ & $0.0531(8)$ & $0.0107(6)$ & $-0.0068(6)$ & $-0.0024(6)$ \\
C53 & $0.0616(10)$ & $0.0562(9)$ & $0.0537(9)$ & $0.0091(7)$ & $-0.0037(7)$ & $0.0016(7)$ \\
C54 & $0.0878(13)$ & $0.0767(12)$ & $0.0519(9)$ & $0.0216(10)$ & $-0.0066(9)$ & $0.0040(9)$ \\
C55 & $0.1021(15)$ & $0.0748(12)$ & $0.0582(11)$ & $0.0315(11)$ & $-0.0277(10)$ & $-0.0137(9)$ \\
C56 & $0.0829(13)$ & $0.0511(9)$ & $0.0773(12)$ & $0.0176(9)$ & $-0.0367(10)$ & $-0.0141(9)$ \\
C57 & $0.0554(9)$ & $0.0389(7)$ & $0.0655(10)$ & $0.0110(6)$ & $-0.0160(7)$ & $-0.0060(7)$ \\
C58 & $0.0548(9)$ & $0.0423(7)$ & $0.0616(9)$ & $-0.0015(6)$ & $0.0010(7)$ & $-0.0032(7)$ \\
N1 & $0.0427(6)$ & $0.0382(6)$ & $0.0447(6)$ & $-0.0008(5)$ & $0.0002(5)$ & $-0.0003(5)$ \\
N2 & $0.0780(10)$ & $0.0480(8)$ & $0.0823(10)$ & $-0.0025(7)$ & $-0.0103(8)$ & $0.0158(7)$ \\
N3 & $0.0606(8)$ & $0.0397(6)$ & $0.0787(10)$ & $-0.0065(6)$ & $-0.0109(7)$ & $-0.0045(6)$ \\
O & $0.0949(10)$ & $0.1018(10)$ & $0.0457(6)$ & $0.0184(8)$ & $-0.0140(6)$ & $-0.0034(6)$ \\
& & & & & & \\
\hline
\end{tabular}

Geometric parameters $\left(\AA,{ }^{\circ}\right)$

\begin{tabular}{llll}
\hline $\mathrm{C} 1-\mathrm{N} 1$ & $1.3370(16)$ & $\mathrm{C} 33-\mathrm{H} 33$ & 0.9300 \\
$\mathrm{C} 1-\mathrm{C} 2$ & $1.3857(18)$ & $\mathrm{C} 34-\mathrm{O}$ & $1.3577(17)$ \\
$\mathrm{C} 1-\mathrm{C} 11$ & $1.4795(17)$ & $\mathrm{C} 34-\mathrm{C} 35$ & $1.375(2)$ \\
$\mathrm{C} 2-\mathrm{C} 3$ & $1.3796(18)$ & $\mathrm{C} 35-\mathrm{C} 36$ & $1.381(2)$ \\
$\mathrm{C} 2-\mathrm{H} 2$ & 0.9300 & $\mathrm{C} 35-\mathrm{H} 35$ & 0.9300 \\
$\mathrm{C} 3-\mathrm{C} 4$ & $1.4035(18)$ & $\mathrm{C} 36-\mathrm{H} 36$ & 0.9300 \\
$\mathrm{C} 3-\mathrm{C} 31$ & $1.4806(18)$ & $\mathrm{C} 37-\mathrm{O}$ & $1.417(2)$ \\
$\mathrm{C} 4-\mathrm{C} 5$ & $1.4069(18)$ & $\mathrm{C} 37-\mathrm{H} 37 \mathrm{~A}$ & 0.9600 \\
$\mathrm{C} 4-\mathrm{C} 41$ & $1.4347(18)$ & $\mathrm{C} 37-\mathrm{H} 37 \mathrm{~B}$ & 0.9600 \\
$\mathrm{C} 5-\mathrm{N} 1$ & $1.3406(16)$ & $\mathrm{C} 37-\mathrm{H} 37 \mathrm{C}$ & 0.9600 \\
$\mathrm{C} 5-\mathrm{C} 51$ & $1.4590(18)$ & $\mathrm{C} 41-\mathrm{N} 2$ & $1.1375(17)$ \\
$\mathrm{C} 11-\mathrm{C} 12$ & $1.3817(18)$ & $\mathrm{C} 51-\mathrm{C} 58$ & $1.3700(19)$ \\
$\mathrm{C} 11-\mathrm{C} 16$ & $1.3833(19)$ & $\mathrm{C} 51-\mathrm{C} 52$ & $1.4368(19)$ \\
$\mathrm{C} 12-\mathrm{C} 13$ & $1.3780(19)$ & $\mathrm{C} 52-\mathrm{C} 53$ & $1.393(2)$ \\
$\mathrm{C} 12-\mathrm{H} 12$ & 0.9300 & $\mathrm{C} 52-\mathrm{C} 57$ & $1.405(2)$ \\
$\mathrm{C} 13-\mathrm{C} 14$ & $1.369(2)$ & $\mathrm{C} 53-\mathrm{C} 54$ & $1.374(2)$ \\
$\mathrm{C} 13-\mathrm{H} 13$ & 0.9300 & $\mathrm{C} 53-\mathrm{H} 53$ & 0.9300 \\
$\mathrm{C} 14-\mathrm{C} 15$ & $1.365(2)$ & $\mathrm{C} 54-\mathrm{C} 55$ & $1.391(3)$ \\
$\mathrm{C} 14-\mathrm{H} 14$ & 0.9300 & $\mathrm{C} 54-\mathrm{H} 54$ & 0.9300 \\
$\mathrm{C} 15-\mathrm{C} 16$ & $1.377(2)$ & $\mathrm{C} 55-\mathrm{C} 56$ & $1.365(3)$ \\
$\mathrm{C} 15-\mathrm{H} 15$ & 0.9300 & $\mathrm{C} 55-\mathrm{H} 55$ & 0.9300 \\
$\mathrm{C} 16-\mathrm{H} 16$ & 0.9300 & $\mathrm{C} 56-\mathrm{C} 57$ & $1.384(2)$ \\
$\mathrm{C} 31-\mathrm{C} 36$ & $1.3771(19)$ & $\mathrm{C} 56-\mathrm{H} 56$ & 0.9300 \\
$\mathrm{C} 31-\mathrm{C} 32$ & $1.3872(19)$ & $\mathrm{C} 57-\mathrm{N} 3$ & $1.367(2)$ \\
$\mathrm{C} 32-\mathrm{C} 33$ & $1.370(2)$ & $\mathrm{C} 58-\mathrm{N} 3$ & $1.3507(19)$ \\
$\mathrm{C} 32-\mathrm{H} 32$ & 0.9300 & $\mathrm{C} 58-\mathrm{H} 58$ & 0.9300 \\
$\mathrm{C} 33-\mathrm{C} 34$ & $1.379(2)$ & $\mathrm{N} 3-\mathrm{H} 3$ & 0.8600 \\
& & &
\end{tabular}




\begin{tabular}{|c|c|c|c|}
\hline $\mathrm{N} 1-\mathrm{C} 1-\mathrm{C} 2$ & $122.03(12)$ & $\mathrm{C} 35-\mathrm{C} 34-\mathrm{C} 33$ & $119.47(13)$ \\
\hline $\mathrm{N} 1-\mathrm{C} 1-\mathrm{C} 11$ & $116.42(11)$ & $\mathrm{C} 34-\mathrm{C} 35-\mathrm{C} 36$ & $119.89(14)$ \\
\hline $\mathrm{C} 2-\mathrm{C} 1-\mathrm{C} 11$ & $121.55(12)$ & $\mathrm{C} 34-\mathrm{C} 35-\mathrm{H} 35$ & 120.1 \\
\hline $\mathrm{C} 3-\mathrm{C} 2-\mathrm{C} 1$ & $120.85(12)$ & $\mathrm{C} 36-\mathrm{C} 35-\mathrm{H} 35$ & 120.1 \\
\hline $\mathrm{C} 3-\mathrm{C} 2-\mathrm{H} 2$ & 119.6 & $\mathrm{C} 31-\mathrm{C} 36-\mathrm{C} 35$ & $121.33(14)$ \\
\hline $\mathrm{C} 1-\mathrm{C} 2-\mathrm{H} 2$ & 119.6 & $\mathrm{C} 31-\mathrm{C} 36-\mathrm{H} 36$ & 119.3 \\
\hline $\mathrm{C} 2-\mathrm{C} 3-\mathrm{C} 4$ & $116.57(12)$ & $\mathrm{C} 35-\mathrm{C} 36-\mathrm{H} 36$ & 119.3 \\
\hline $\mathrm{C} 2-\mathrm{C} 3-\mathrm{C} 31$ & $119.10(12)$ & $\mathrm{O}-\mathrm{C} 37-\mathrm{H} 37 \mathrm{~A}$ & 109.5 \\
\hline $\mathrm{C} 4-\mathrm{C} 3-\mathrm{C} 31$ & $124.31(11)$ & $\mathrm{O}-\mathrm{C} 37-\mathrm{H} 37 \mathrm{~B}$ & 109.5 \\
\hline $\mathrm{C} 3-\mathrm{C} 4-\mathrm{C} 5$ & $119.94(11)$ & $\mathrm{H} 37 \mathrm{~A}-\mathrm{C} 37-\mathrm{H} 37 \mathrm{~B}$ & 109.5 \\
\hline $\mathrm{C} 3-\mathrm{C} 4-\mathrm{C} 41$ & $120.15(12)$ & $\mathrm{O}-\mathrm{C} 37-\mathrm{H} 37 \mathrm{C}$ & 109.5 \\
\hline $\mathrm{C} 5-\mathrm{C} 4-\mathrm{C} 41$ & $119.91(12)$ & $\mathrm{H} 37 \mathrm{~A}-\mathrm{C} 37-\mathrm{H} 37 \mathrm{C}$ & 109.5 \\
\hline $\mathrm{N} 1-\mathrm{C} 5-\mathrm{C} 4$ & $121.15(12)$ & $\mathrm{H} 37 \mathrm{~B}-\mathrm{C} 37-\mathrm{H} 37 \mathrm{C}$ & 109.5 \\
\hline $\mathrm{N} 1-\mathrm{C} 5-\mathrm{C} 51$ & $114.99(12)$ & $\mathrm{N} 2-\mathrm{C} 41-\mathrm{C} 4$ & $177.55(17)$ \\
\hline $\mathrm{C} 4-\mathrm{C} 5-\mathrm{C} 51$ & $123.85(12)$ & $\mathrm{C} 58-\mathrm{C} 51-\mathrm{C} 52$ & $106.12(12)$ \\
\hline $\mathrm{C} 12-\mathrm{C} 11-\mathrm{C} 16$ & $118.24(12)$ & $\mathrm{C} 58-\mathrm{C} 51-\mathrm{C} 5$ & $127.06(13)$ \\
\hline $\mathrm{C} 12-\mathrm{C} 11-\mathrm{C} 1$ & $120.66(12)$ & $\mathrm{C} 52-\mathrm{C} 51-\mathrm{C} 5$ & $126.44(12)$ \\
\hline $\mathrm{C} 16-\mathrm{C} 11-\mathrm{C} 1$ & $121.11(12)$ & $\mathrm{C} 53-\mathrm{C} 52-\mathrm{C} 57$ & $118.51(14)$ \\
\hline $\mathrm{C} 13-\mathrm{C} 12-\mathrm{C} 11$ & $120.40(13)$ & $\mathrm{C} 53-\mathrm{C} 52-\mathrm{C} 51$ & $134.80(14)$ \\
\hline $\mathrm{C} 13-\mathrm{C} 12-\mathrm{H} 12$ & 119.8 & $\mathrm{C} 57-\mathrm{C} 52-\mathrm{C} 51$ & $106.67(13)$ \\
\hline $\mathrm{C} 11-\mathrm{C} 12-\mathrm{H} 12$ & 119.8 & $\mathrm{C} 54-\mathrm{C} 53-\mathrm{C} 52$ & $118.99(16)$ \\
\hline $\mathrm{C} 14-\mathrm{C} 13-\mathrm{C} 12$ & $120.70(14)$ & $\mathrm{C} 54-\mathrm{C} 53-\mathrm{H} 53$ & 120.5 \\
\hline $\mathrm{C} 14-\mathrm{C} 13-\mathrm{H} 13$ & 119.6 & $\mathrm{C} 52-\mathrm{C} 53-\mathrm{H} 53$ & 120.5 \\
\hline $\mathrm{C} 12-\mathrm{C} 13-\mathrm{H} 13$ & 119.6 & $\mathrm{C} 53-\mathrm{C} 54-\mathrm{C} 55$ & $121.26(18)$ \\
\hline $\mathrm{C} 15-\mathrm{C} 14-\mathrm{C} 13$ & $119.45(14)$ & $\mathrm{C} 53-\mathrm{C} 54-\mathrm{H} 54$ & 119.4 \\
\hline $\mathrm{C} 15-\mathrm{C} 14-\mathrm{H} 14$ & 120.3 & $\mathrm{C} 55-\mathrm{C} 54-\mathrm{H} 54$ & 119.4 \\
\hline $\mathrm{C} 13-\mathrm{C} 14-\mathrm{H} 14$ & 120.3 & $\mathrm{C} 56-\mathrm{C} 55-\mathrm{C} 54$ & $121.20(16)$ \\
\hline $\mathrm{C} 14-\mathrm{C} 15-\mathrm{C} 16$ & $120.36(15)$ & $\mathrm{C} 56-\mathrm{C} 55-\mathrm{H} 55$ & 119.4 \\
\hline $\mathrm{C} 14-\mathrm{C} 15-\mathrm{H} 15$ & 119.8 & $\mathrm{C} 54-\mathrm{C} 55-\mathrm{H} 55$ & 119.4 \\
\hline $\mathrm{C} 16-\mathrm{C} 15-\mathrm{H} 15$ & 119.8 & $\mathrm{C} 55-\mathrm{C} 56-\mathrm{C} 57$ & $117.69(17)$ \\
\hline $\mathrm{C} 15-\mathrm{C} 16-\mathrm{C} 11$ & 120.85 & $\mathrm{C} 55-\mathrm{C} 56-\mathrm{H} 56$ & 121.2 \\
\hline $\mathrm{C} 15-\mathrm{C} 16-\mathrm{H} 16$ & 119.6 & $\mathrm{C} 57-\mathrm{C} 56-\mathrm{H} 56$ & 121.2 \\
\hline $\mathrm{C} 11-\mathrm{C} 16-\mathrm{H} 16$ & 119.6 & $\mathrm{~N} 3-\mathrm{C} 57-\mathrm{C} 56$ & $130.11(16)$ \\
\hline $\mathrm{C} 36-\mathrm{C} 31-\mathrm{C} 32$ & $117.89(13)$ & $\mathrm{N} 3-\mathrm{C} 57-\mathrm{C} 52$ & $107.52(13)$ \\
\hline $\mathrm{C} 36-\mathrm{C} 31-\mathrm{C} 3$ & $123.59(12)$ & $\mathrm{C} 56-\mathrm{C} 57-\mathrm{C} 52$ & $122.35(16)$ \\
\hline $\mathrm{C} 32-\mathrm{C} 31-\mathrm{C} 3$ & $118.50(12)$ & $\mathrm{N} 3-\mathrm{C} 58-\mathrm{C} 51$ & $110.08(14)$ \\
\hline $\mathrm{C} 33-\mathrm{C} 32-\mathrm{C} 31$ & $121.22(13)$ & $\mathrm{N} 3-\mathrm{C} 58-\mathrm{H} 58$ & 125.0 \\
\hline $\mathrm{C} 33-\mathrm{C} 32-\mathrm{H} 32$ & 119.4 & $\mathrm{C} 51-\mathrm{C} 58-\mathrm{H} 58$ & 125.0 \\
\hline $\mathrm{C} 31-\mathrm{C} 32-\mathrm{H} 32$ & 119.4 & $\mathrm{C} 1-\mathrm{N} 1-\mathrm{C} 5$ & $119.08(11)$ \\
\hline $\mathrm{C} 32-\mathrm{C} 33-\mathrm{C} 34$ & $120.19(14)$ & $\mathrm{C} 58-\mathrm{N} 3-\mathrm{C} 57$ & $109.59(13)$ \\
\hline $\mathrm{C} 32-\mathrm{C} 33-\mathrm{H} 33$ & 119.9 & $\mathrm{C} 58-\mathrm{N} 3-\mathrm{H} 3$ & 125.2 \\
\hline $\mathrm{C} 34-\mathrm{C} 33-\mathrm{H} 33$ & 119.9 & $\mathrm{C} 57-\mathrm{N} 3-\mathrm{H} 3$ & 125.2 \\
\hline $\mathrm{O}-\mathrm{C} 34-\mathrm{C} 35$ & $125.05(14)$ & $\mathrm{C} 34-\mathrm{O}-\mathrm{C} 37$ & $118.26(14)$ \\
\hline $\mathrm{O}-\mathrm{C} 34-\mathrm{C} 33$ & $115.48(14)$ & & \\
\hline $\mathrm{N} 1-\mathrm{C} 1-\mathrm{C} 2-\mathrm{C} 3$ & $5.0(2)$ & $\mathrm{C} 33-\mathrm{C} 34-\mathrm{C} 35-\mathrm{C} 36$ & $0.0(3)$ \\
\hline $\mathrm{C} 11-\mathrm{C} 1-\mathrm{C} 2-\mathrm{C} 3$ & $-175.75(12)$ & $\mathrm{C} 32-\mathrm{C} 31-\mathrm{C} 36-\mathrm{C} 35$ & $-0.4(2)$ \\
\hline $\mathrm{C} 1-\mathrm{C} 2-\mathrm{C} 3-\mathrm{C} 4$ & $-1.04(19)$ & $\mathrm{C} 3-\mathrm{C} 31-\mathrm{C} 36-\mathrm{C} 35$ & $178.13(14)$ \\
\hline
\end{tabular}




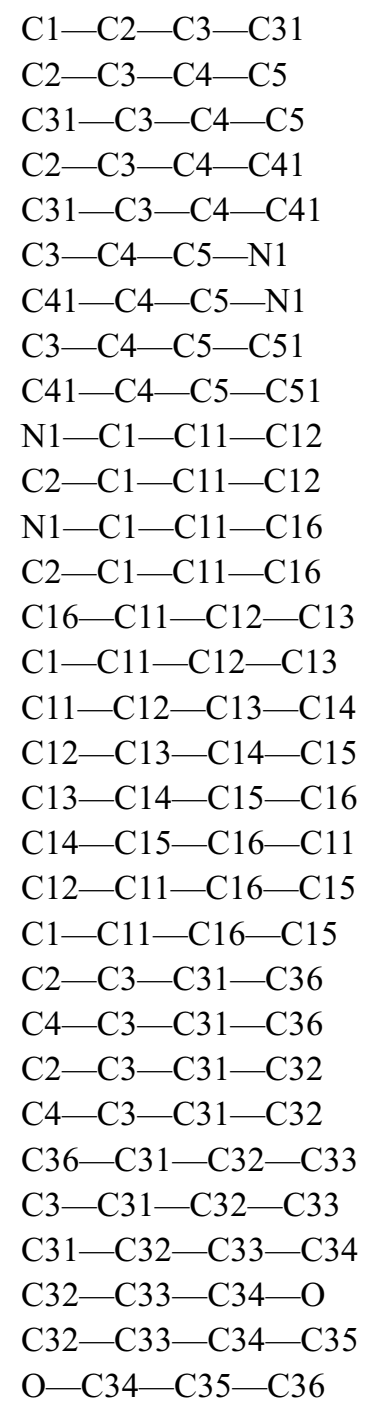

$-179.38(12)$

$-4.40(19)$

$173.84(12)$

$175.41(13)$

$-6.3(2)$

$6.38(19)$

$-173.43(12)$

$-172.52(12)$

7.7 (2)

24.87 (18)

$-154.39(13)$

$-154.96(14)$

25.8 (2)

-0.5 (2)

179.65 (13)

$0.6(2)$

$-0.6(3)$

0.4 (3)

$-0.3(3)$

$0.3(2)$

$-179.83(15)$

$-132.13(15)$

49.7 (2)

46.38 (19)

$-131.82(14)$

-0.2 (2)

$-178.80(14)$

0.7 (2)

$179.74(15)$

$-0.6(3)$

179.65 (16)

$$
\begin{aligned}
& \text { C34-C35-C36-C31 } \\
& \mathrm{N} 1-\mathrm{C} 5-\mathrm{C} 51-\mathrm{C} 58 \\
& \mathrm{C} 4-\mathrm{C} 5-\mathrm{C} 51-\mathrm{C} 58 \\
& \mathrm{~N} 1-\mathrm{C} 5-\mathrm{C} 51-\mathrm{C} 52 \\
& \mathrm{C} 4-\mathrm{C} 5-\mathrm{C} 51-\mathrm{C} 52 \\
& \mathrm{C} 58-\mathrm{C} 51-\mathrm{C} 52-\mathrm{C} 53 \\
& \mathrm{C} 5-\mathrm{C} 51-\mathrm{C} 52-\mathrm{C} 53 \\
& \text { C58-C51-C52-C57 } \\
& \mathrm{C} 5-\mathrm{C} 51-\mathrm{C} 52-\mathrm{C} 57 \\
& \mathrm{C} 57-\mathrm{C} 52-\mathrm{C} 53-\mathrm{C} 54 \\
& \mathrm{C} 51-\mathrm{C} 52-\mathrm{C} 53-\mathrm{C} 54 \\
& \mathrm{C} 52-\mathrm{C} 53-\mathrm{C} 54-\mathrm{C} 55 \\
& \mathrm{C} 53-\mathrm{C} 54-\mathrm{C} 55-\mathrm{C} 56 \\
& \mathrm{C} 54-\mathrm{C} 55-\mathrm{C} 56-\mathrm{C} 57 \\
& \mathrm{C} 55-\mathrm{C} 56-\mathrm{C} 57-\mathrm{N} 3 \\
& \mathrm{C} 55-\mathrm{C} 56-\mathrm{C} 57-\mathrm{C} 52 \\
& \mathrm{C} 53-\mathrm{C} 52-\mathrm{C} 57-\mathrm{N} 3 \\
& \mathrm{C} 51-\mathrm{C} 52-\mathrm{C} 57-\mathrm{N} 3 \\
& \mathrm{C} 53-\mathrm{C} 52-\mathrm{C} 57-\mathrm{C} 56 \\
& \mathrm{C} 51-\mathrm{C} 52-\mathrm{C} 57-\mathrm{C} 56 \\
& \mathrm{C} 52-\mathrm{C} 51-\mathrm{C} 58-\mathrm{N} 3 \\
& \mathrm{C} 5-\mathrm{C} 51-\mathrm{C} 58-\mathrm{N} 3 \\
& \mathrm{C} 2-\mathrm{C} 1-\mathrm{N} 1-\mathrm{C} 5 \\
& \mathrm{C} 11-\mathrm{C} 1-\mathrm{N} 1-\mathrm{C} 5 \\
& \mathrm{C} 4-\mathrm{C} 5-\mathrm{N} 1-\mathrm{C} 1 \\
& \mathrm{C} 51-\mathrm{C} 5-\mathrm{N} 1-\mathrm{C} 1 \\
& \mathrm{C} 51-\mathrm{C} 58-\mathrm{N} 3-\mathrm{C} 57 \\
& \mathrm{C} 56-\mathrm{C} 57-\mathrm{N} 3-\mathrm{C} 58 \\
& \mathrm{C} 52-\mathrm{C} 57-\mathrm{N} 3-\mathrm{C} 58 \\
& \mathrm{C} 35-\mathrm{C} 34-\mathrm{O}-\mathrm{C} 37 \\
& \mathrm{C} 33-\mathrm{C} 34-\mathrm{O}-\mathrm{C} 37
\end{aligned}
$$

0.5 (3)

$-143.80(14)$

$35.2(2)$

28.20 (19)

-152.85 (13)

$-178.06(16)$

$8.6(3)$

$0.18(15)$

-173.18 (13)

$-0.6(2)$

177.47 (15)

0.1 (2)

0.3 (3)

$-0.2(3)$

$-178.46(16)$

-0.3 (2)

$179.24(13)$

$0.66(15)$

0.7 (2)

$-177.84(14)$

-0.98 (16)

$172.33(13)$

-3.18 (19)

177.56 (11)

$-2.49(18)$

176.49 (11)

1.44 (17)

$177.06(16)$

-1.28 (16)

$-6.1(3)$

173.55 (16)

Hydrogen-bond geometry $\left(A,{ }^{\circ}\right)$

$\mathrm{Cg} 1$ is the centroid of the pyrrole ring.

\begin{tabular}{lllll}
\hline$D-\mathrm{H} \cdots A$ & $D-\mathrm{H}$ & $\mathrm{H} \cdots A$ & $D \cdots A$ & $D-\mathrm{H} \cdots A$ \\
\hline $\mathrm{N} 3-\mathrm{H} 3 \cdots \mathrm{N} 2^{\mathrm{i}}$ & 0.86 & 2.15 & $2.9693(19)$ & 159 \\
$\mathrm{C} 32-\mathrm{H} 32 \cdots C g 1^{1 i}$ & 0.93 & 3.00 & $3.9157(19)$ & 170 \\
\hline
\end{tabular}

Symmetry codes: (i) $-x,-y+1,-z+2$; (ii) $-x+1,-y+1,-z$. 